\title{
Comparison of natural cycles versus hormone replacement treatment cycles for endometrial preparation prior to frozen-thawed embryo transfer: a retrospective cohort study from 9733 cycles
}

\author{
Xitong Liu ${ }^{1}$, Hui Wang ${ }^{1}, \mathrm{He} \mathrm{Cai}^{1}$, and Juanzi Shi ${ }^{1}$ \\ ${ }^{1}$ Affiliation not available
}

November 6, 2020

\begin{abstract}
Objective To compare the effectiveness of natural cycles (NC) and artificial cycles (AC) in women undergoing frozen-thawed embryo transfer (FET) after in vitro fertilization (IVF). Design Retrospective cohort study. Setting Public fertility center in China. Population We studied 9733 women undergoing the first cycle of FET over a 3-year period (June 2014 - December 2017 ). All women were followed-up until one year after embryo transfer. Methods The type of endometrial preparation was determined by the treating physician's preference, based on patients' characteristics. Women with regular ovulation were allocated to natural cycles $(n=1676)$, while patients who were reluctant to frequently monitoring or living far from the hospital were allocated to artificial cycles $(\mathrm{n}=8057)$. A logistic regression model was used to assess the association between endometrial preparation and clinical outcomes while adjusting for potential confounders. Main outcome measures Live-birth rate was primary outcome while miscarriage rate, clinical pregnancy rate, preterm birth rate, and ectopic pregnancy rate were secondary outcomes. Results In the adjusted model, type of endometrial preparation did not affect live birth (OR, 0.89; 95\%CI, 0.79-1.01), clinical pregnancy (OR, 0.96; 95\%CI, 0.85-1.09), preterm birth (OR, 1.09; 95\%CI, 0.90-1.33) and ectopic pregnancy (OR, 0.77; 95\%CI, 0.36-1.61), while AC significantly increased the miscarriage rate (OR, 1.38; 95\%CI, 1.11-1.73, P=0.004). Conclusion In women undergoing FET, natural cycles and artificial cycles resulted in comparable live birth rate while miscarriage rate was higher in artificial cycles.
\end{abstract}

Comparison of natural cycles versus hormone replacement treatment cycles for endometrial preparation prior to frozen-thawed embryo transfer: a retrospective cohort study from 9733 cycles

Xitong Liu, ${ }^{a}$ Hui Wang, ${ }^{a}$ He Cai, ${ }^{a}$ Juanzi Shi, ${ }^{\text {a }}$

a The Assisted Reproduction Center, Northwest women's and Children's Hospital, Xi'an, China

Correspondence: Juanzi Shi, The Assisted Reproduction Center, Northwest women's and Children's Hospital, Xi'an, China.

Email: shijuanzi123@163.com

Running title: Outcome of NC vs HRT

Objective To compare the effectiveness of natural cycles (NC) and artificial cycles (AC) in women undergoing frozen-thawed embryo transfer (FET) after in vitro fertilization (IVF).

Design Retrospective cohort study.

Setting Public fertility center in China. 
Population We studied 9733 women undergoing the first cycle of FET over a 3-year period (June 2014 December 2017). All women were followed-up until one year after embryo transfer.

Methods The type of endometrial preparation was determined by the treating physician's preference, based on patients' characteristics. Women with regular ovulation were allocated to natural cycles $(\mathrm{n}=1676)$, while patients who were reluctant to frequently monitoring or living far from the hospital were allocated to artificial cycles $(n=8057)$. A logistic regression model was used to assess the association between endometrial preparation and clinical outcomes while adjusting for potential confounders.

Main outcome measures Live-birth rate was primary outcome while miscarriage rate, clinical pregnancy rate, preterm birth rate, and ectopic pregnancy rate were secondary outcomes.

$\mathbf{R}$ esults In the adjusted model, type of endometrial preparation did not affect live birth (OR, 0.89; 95\%CI, 0.79-1.01), clinical pregnancy (OR, 0.96; 95\%CI, 0.85-1.09), preterm birth (OR, 1.09; 95\% CI, 0.90-1.33) and ectopic pregnancy (OR, 0.77; 95\% CI, 0.36-1.61), while AC significantly increased the miscarriage rate (OR, $1.38 ; 95 \% \mathrm{CI}, 1.11-1.73, \mathrm{P}=0.004)$.

Conclusion In women undergoing FET, natural cycles and artificial cycles resulted in comparable live birth rate while miscarriage rate was higher in artificial cycles.

Keywords frozen-thawed embryo transfer, natural cycles, artificial cycles, miscarriage rate, live birth rate.

Tweetable abstract In women undergoing FET, natural cycles and artificial cycles resulted in comparable live birth rate while miscarriage rate was higher in artificial cycles.

\section{Introduction}

With the introduction of more efficient cryopreservation techniques, and an increasing number of studies claim better results from frozen-thawed embryo transfer (FET) compared to fresh embryo transfer ${ }^{1-3}$. As a consequence, FET cycles are on the increase worldwide.

Embryo implantation is a critical step affecting clinical outcomes of in vitro fertilization (IVF). The optimal endometrial preparation for FET is still under discussion. Endometrial preparation can be done in the natural cycles $(\mathrm{NC})$ or with artificial cycles $(\mathrm{AC})$.

A Cochrane review published in 2017 suggested a comparable clinical pregnancy rate (OR 1.06; 95\% CI, 0.40-2.80) of different types of endometrial preparation. The quality of the underlying studies was rated as very $\mathrm{low}^{4}$, while live birth and miscarriage rates were not reported. Some studies have shown similar live birth rates and miscarriage rates between $\mathrm{NC}$ and $\mathrm{AC}^{5,6}$. In contrast, other studies have reported increased miscarriage rates and lower live birth rates in $\mathrm{AC}$ than $\mathrm{NC}^{7}$. In view of these conflicting data, we compared live birth rates and other clinical outcomes following FET conducted using natural cycle to those in which endometrial preparation was achieved via artificial cycles.

\section{Methods}

\section{Study design and patients}

We performed a retrospective cohort study between June 2014 and December 2017 in the Center for Assisted Reproductive Technology of Northwest Women's and Children's Hospital, Xi'an, China. All patients were followed-up for one year. This study was approved by the ethics committee of the hospital (number 2018002). As part of the clinical process, all patients signed informed consent before the IVF/FET procedure. Data was extracted from our electronic medical record system.

Women who underwent a first cycle of FET were eligible for the study. Women with polycystic ovarian syndrome (PCOS) or other forms of anovulation, and women in whom the thawed embryo did not survive in the current FET cycle were excluded.

\section{Endometrial preparation protocols}


In this study, different methods of endometrial preparation methods were non-selectively and consecutively recorded, and used exposure variables to observe the correlation with live birth rate and other clinical outcomes. The type of endometrial preparation was determined by the treating physician's preference, based on patients' characteristics. In general, women with regular ovulation were allocated to natural cycles $(\mathrm{n}=1676)$, while patients who were reluctant to frequently monitoring or living far from the hospital were allocated to artificial cycles $(\mathrm{n}=8057)$. The detailed protocols for $\mathrm{NC}$ and $\mathrm{AC}$ endometrial preparation were described as follows:

1) Natural cycles for FET

Follicle monitoring began on day 8-10 of the menstrual cycle. When the leading follicle reached a mean diameter of $>17 \mathrm{~mm}$, serum luteinizing hormone (LH) was $<20 \mathrm{IU} / \mathrm{L}, 10000 \mathrm{IU}$ of human chorionic gonadotropin (hCG) was administered to trigger oocytes ovulation. Ovulation was confirmed by transvaginal ultrasound the day after $\mathrm{hCG}$ and the next day. When $\mathrm{LH}$ was $>20 \mathrm{IU} / \mathrm{L}$, transvaginal ultrasound was performed every day until ovulation.

Artificial cycles for FET

In women treated with AC, endometrial priming started on the fifth day of the menstrual cycle with estradiol valerate (Progynova; Bayer Schering Pharma AG, Berlin, Germany) orally administered at a dose of 6mg daily. After 10-12 days of endometrial preparation, transvaginal ultrasound and progesterone level were performed. In women with endometrial thickness $>8 \mathrm{~mm}$ and serum progesterone $(\mathrm{P})$ level $<1.5 \mathrm{ng} / \mathrm{ml}$, intramuscular progesterone at a dose of $60 \mathrm{mg}$ daily was administrated.

The timing of FET was based on the day of embryo freezing and the day of ovulation (i.e., 3 days after ovulation for cleavage stage embryos and 5 days after ovulation for blastocyst stage embryos). Triple-line endometrial pattern of endometrium was classified as pattern A (a triple-line pattern consisting of a central hyperechoic line surrounded by two hypoechoic layers), pattern B (an intermediate isoechogenic pattern with the same reflectivity as the surrounding myometrium and a poorly defined central echogenic line), and pattern $\mathrm{C}$ (homogenous, hyperechogenic endometrium) ${ }^{8}$.

\section{Luteal phase support}

$60 \mathrm{mg}$ of intramuscular progesterone (Progesterone injection; Xianju, Zhejiang, China), 20-30mg oral progesterone (Dydrogesterone; Abbott Biologicals B.V., The Netherlands) daily were given from the day of embryo transfer (ET) in both $\mathrm{NC}$ and $\mathrm{AC}$ groups. All women were followed up and pregnancy test with biochemical hCG was performed 14 days after ET. If serum hCG >50 IU/L, luteal support continued. Transvaginal ultrasound was performed 5 weeks after embryo transfer to determine the number of gestational sacs and fetal viability.

\section{Outcome measures}

According to ASRM consensus and ACOG, the definition of clinical outcomes was confirmed ${ }^{9,10}$. Our main outcome was live birth while miscarriage rate, clinical pregnancy rate, preterm birth rate, and ectopic pregnancy rate were secondary outcomes. Live birth was defined as the delivery of at least one live-born baby beyond 22 weeks of gestation. Live birth rate was defined as live deliveries (at least one live birth) per woman after embryo transfer. Miscarriage rate was defined as the spontaneous loss of a clinical pregnancy before 22 completed weeks of gestational age per clinical pregnancy cycle. Clinical pregnancy was defined as a pregnancy diagnosed by ultrasonographic visualization of one or more gestational sacs or definitive clinical signs of pregnancy. Preterm birth was defined as a birth that takes place after 22 weeks and before 37 completed weeks of gestational age. Ectopic pregnancy was defined as gestational sac observed by ultrasound outside the uterine cavity. Implantation rate was defined as the number of gestational sacs observed divided by the number of embryos transferred.

\section{Statistical analysis}


The descriptive data on participant characteristics are summarized using the mean and standard deviation for continuous variables. Counts and proportions are used for the categorical variables. Chi-square tests were performed to compare the categorical variables. To compare the continuous variables among the groups, an analysis of covariance (normally distributed variables) or a Kruskal-Wallis test (abnormally distributed variables) were used. For multivariate analyses, logistic regression was utilized. Crude odds ratios (OR) and adjusted ORs (aOR) with 95\% confidence interval (CI) were calculated to assess the association between covariates and clinical outcomes. We selected the covariates on the basis of their associations with the outcomes of interest or a change in effect estimate of more than $10 \%$. In the adjusted Model: we adjusted female age at embryo transfer, infertility duration, BMI, AFC, no. of oocyte retrieved, infertility type, protocol in the fresh cycle, single blastocyst transfer, endometrial thickness on the day of $\mathrm{P}$ administration, triple-line endometrial pattern, type of embryo transferred, no. of transferred embryos, no. of transferrable embryo and no. of good quality embryos transferred.

Interaction and stratified analyses were conducted according to female age at oocyte retrieval, female age at embryo transfer, infertility duration, infertility type, AFC, endometrial thickness on the day of $\mathrm{P}$ administration, fertilization type, protocol in the fresh cycle, no. of oocyte retrieved, no. of transferrable embryos, no. of transferred embryos, no. of good quality embryos transferred, type of embryo transferred, triple-line endometrial pattern and single blastocyst transfer. Data were analyzed with the use of the statistical packages R (The R Foundation; http://www.r-project.org;version 3.4.3) and Empower (R) (www.empowerstates.com, $\mathrm{X} \& \mathrm{Y}$ solutions, inc. Boston, Massachusetts). The level of significance was set at $p<0.05$. The type I error rate was controlled using the Bonferroni stepdown procedure for multiple comparisons. When Bonferroni corrections were applied, unadjusted $p=0.05$; the number of tests, 5 (live birth rate, miscarriage rate, clinical pregnancy rate, preterm birth rate, ectopic pregnancy rate); $p=0.01$ with Bonferroni correction applied $[0.05 \div 5])$.

\section{Results}

We studied 9733 women, 1676 in the NC group and 8057 in the AC group. Baseline characteristics are presented in Table 1. Compared to women in the NC group, women in the AC group were younger, had higher BMI, a higher AFC, more no. of oocyte retrieved, and used more often an agonist protocol in the fresh cycle. They had thinner endometrium on the day of $\mathrm{P}$ administration and less often a triple-line pattern, more transferrable embryos and more good quality embryos transferred. Table 2 shows the pregnancy outcomes in $\mathrm{NC}$ and $\mathrm{AC}$. Women in the AC group had a higher implantation rate, miscarriage and more male baby born compared with women in NC.

Univariate analysis was performed to evaluate the effect of each variable on the live birth rate (Table 3 ). Female age at oocyte retrieval and embryo transfer, BMI, infertility duration, secondary infertility, antagonist and other protocol in the fresh cycle, triple-line endometrial pattern $(\mathrm{C})$ and single blastocyst transfer were negatively associated with live birth rate, while AFC, endometrial thickness on the day of $\mathrm{P}$ administration, no. of oocyte retrieved, no. of transferrable embryo, no. of good quality embryos transferred and blastocyst embryo transfer were positively associated with live birth rate.

A logistic regression model was then used to assess the association between endometrial preparation and clinical outcomes while adjusting for potential confounders presented in Table 4 . In the adjusted model, type of endometrial preparation did not affect live birth (OR, 0.89; 95\% CI, 0.79-1.01), clinical pregnancy (OR, $0.96 ; 95 \% \mathrm{CI}, 0.85-1.09)$, preterm birth (OR, 1.09; 95\% CI, 0.90-1.33) and ectopic pregnancy (OR, 0.77; 95\%CI, 0.36-1.61), while AC significantly increased the miscarriage rate (OR, 1.38; 95\%CI, 1.11-1.73, $\mathrm{P}=0.004)$.

Interaction and stratified analyses were shown in Table S1 and Table S2. Live birth rates were comparable in all subgroups. Higher miscarriage rate was observed in patients with AC in all subgroups of female age at oocyte retrieval, female age at embryo transfer, infertility duration, infertility type, AFC, endometrial thickness on the day of $\mathrm{P}$ administration, fertilization type, protocol in the fresh cycle, no. of oocyte retrieved, no. of transferrable embryo, no. of transferred embryos, no. of good quality embryos transferred, type of embryo transferred, triple-line endometrial pattern and single blastocyst transfer. There were no significant 
interactions in any of the subgroups ( $p>0.05$ for all comparisons).

\section{Discussion}

\section{Main findings}

We found no statistically significant difference in the live birth rate between $\mathrm{NC}$ and $\mathrm{AC}$ for endometrial preparation. Our results are in line with those of other studies in recent literature ${ }^{11-13}$. Our study of no difference between $\mathrm{NC}$ and $\mathrm{AC}$ contrasts with studies that found a higher live birth rate with $\mathrm{AC}^{14,15}$. However, these studies relied on home ovulation predictor kits in $\mathrm{NC}$ or without serum hormone analysis in $\mathrm{AC}$ cycles and may be less precise in evaluating ovulation. In addition, the sample size was quite small.

Our study demonstrated a higher miscarriage rate in AC groups. These findings are similar to results from previous studies. A prospective study of 570 cycles found a higher miscarriage rate in the AC group but only included young women with regular menstrual $\mathrm{cycles}^{16}$. A retrospective study of 1677 FET cycles found that the miscarriage rate was significantly higher in the AC group both in own-egg and ovum-donor patients ${ }^{17}$.

Our findings contrast with several other retrospective studies showing similar miscarriage rates between NC and AC. One retrospective cohort study reported a comparable miscarriage rate between $\mathrm{NC}$ and $\mathrm{AC}$ after adjusting for potential confounders, while the AC group harms obstetric outcome ${ }^{7}$. Although this study had a relatively larger sample size, it was limited in its inclusion criteria of regular ovulatory cycles and at most two previous ET cycles.

\section{Strengths and limitations}

Our present work has some strength of note. Firstly, we adjusted more variables to ensure that the result was more reliable. Secondly, proper statistical methods and sensitivity analysis ensure structural stability. Thirdly, the study included solely ovulatory women to cleanly analyze the impact of both types of endometrial preparation. Last but not least, we only included women in the first cycle of FET, thus avoiding bias from multiple cycles.

Several limitations are associated with the present study warrant mention. Firstly, the type of endometrial preparation was chosen by the treating physician's preference and this may introduce a potential bias. Secondly, this was a retrospective study and could not investigate other confounders, including exercises, nutritional supplements and diets. Thirdly, we could not compare the incidence of preeclampsia and placental dysfunction in pregnancies after NC and AC, since these data were not collected in our dataset.

\section{Interpretation}

Until now, it remains unclear if estradiol valerate and progesterone harm endometrial receptivity and embryo development. The adverse clinical outcome of AC might be caused by supraphysiological hormone levels during early trophoblast invasion, which could lead to abnormal pregnancy ${ }^{18-20}$. Estradiol is essential for endometrial and placental development. One historical cohort study indicated elevated serum estradiol levels in $\mathrm{AC}$ cycles are associated with lower ongoing pregnancy rate and live birth rate ${ }^{21}$. Excess estradiol levels in the early stages of pregnancy can have adverse effects on placentation and may in turn cause miscarriage. In vitro study has shown estradiol can cause cell death and inhibit trophoblast invasion in both first-trimester human cytotrophoblast cell line and placental explants ${ }^{22}$. Studies have indicated preeclampsia in pregnancy is associated with $\mathrm{FET}^{23,24}$. Unfortunately, we have no data on pre-eclampsia. One retrospective study suggested a higher risk of hypertension disorder in women of both singletons and multiples after AC than $\mathrm{NC}^{7}$. The adverse obstetric outcomes of AC for endometrial preparation indicate a possible link between endometrial preparation and placenta-related diseases, which might be caused by supraphysiological hormone levels during early trophoblast invasion. In natural cycles, corpus luteum (CL) is derived from the ovulated follicle. The formation of CL is indispensable for steroid hormone production required to support endometrial receptivity for successful implantation and maintenance of early pregnancy. However, women in the AC cycle do not have CL to produce hormone that is necessary to sustain early pregnancy. Instead, women in the $\mathrm{AC}$ cycle were given exogenous estradiol and progesterone and maybe too much or less for individual need. 
Exogenous endometrial preparation may decrease the ability of embryos selecting the endometrium, which could, in turn, lead to abnormal embryos implantation and miscarriage.

Although we did not find a difference in the live birth rate between two protocols, we did demonstrate a higher miscarriage rate in AC cycles. Miscarriage is a heartbreaking experience for patients and may lead to intrauterine infection or adhesion which could, in turn, have a negative effect on embryo implantation for the next cycle of embryo transfer. Even though AC is convenient for both doctors and patients, a higher miscarriage rate should be considered before making decisions.

\section{Conclusion}

In women undergoing FET, natural cycles (NC) and hormone replacement cycles (AC) resulted in comparable live birth rate while miscarriage rate was higher in hormone replacement cycles. Further multi-center randomized clinical trials are needed to confirm the relationship between $\mathrm{AC}$ and miscarriage.

Disclosure of interests

None declared.

Contribution to authorship

XL designed the study. HW and HC conducted data collection, analysis and writing the manuscript. JS conceived and designed the study.

\section{Details of ethics approval}

This study was approved by the ethics committee of the hospital (number 2018002).

\section{Funding}

Supported by National Natural Science Foundation of China (No. 81771657 http://www.nsfc.gov.cn/) and General Projects of Social Development in Shaanxi Province(No. 2018SF-260).

\section{Acknowledgements}

The authors thank the staff from Northwest Women's and Children's Hospital for their assistance with the data collection.

\section{$R$ eferences}

1. Zhu, D, Zhang J, Cao S, et al. Vitrified-warmed blastocyst transfer cycles yield higher pregnancy and implantation rates compared with fresh blastocyst transfer cycles-time for a new embryo transfer strategy? Fertil Steril. 2011; 95(5): p. 1691-5.

2. Loutradi, K.E, Kolibianakis EM, Venetis CA, et al. Cryopreservation of human embryos by vitrification or slow freezing: a systematic review and meta-analysis. Fertil Steril. 2008; 90(1): p. 186-93.

3. Belva, F, Bonduelle M, Roelants M, et al. Neonatal health including congenital malformation risk of 1072 children born after vitrified embryo transfer. Hum Reprod. 2016; 31(7): p. 1610-20.

4. Ghobara T, Gelbaya TA, Ayeleke RO. Cycle regimens for frozen-thawed embryo transfer. The Cochrane database of systematic reviews. 2017; 7(7): Cd003414.

5. Lathi, R.B, Chi YY, et al. Frozen blastocyst embryo transfer using a supplemented natural cycle protocol has a similar live birth rate compared to a programmed cycle protocol. J Assist Reprod Genet. 2015; 32(7): p. 1057-62.

6. Agha-Hosseini, M, Hashemi L, Aleyasin A, et al. Natural cycle versus artificial cycle in frozen-thawed embryo transfer: A randomized prospective trial. Turk J Obstet Gynecol. 2018; 15(1): p. 12-17.

7. Jing, S, Li XF, Zhang S, et al. Increased pregnancy complications following frozen-thawed embryo transfer during an artificial cycle. J Assist Reprod Genet. 2019; 36(5): p. 925-933.

8. Zhao J, Zhang Q, Wang Y, et al. Endometrial pattern, thickness and growth in predicting pregnancy outcome following 3319 ivf cycle. Reproductive biomedicine online 2014; 29(3): 291-298. 
9. Zegers-Hochschild, F, Adamson GD, Dyer S, et al. The International Glossary on Infertility and Fertility Care, 2017. Hum Reprod 2017; 32(9): p. 1786-1801.

10. American College of O, Gynecologists' Committee on Practice BO. Practice Bulletin No. 171: Management of Preterm Labor. Obstet Gynecol 2016; 128(10): e155-e164.

11. Gelbaya TA, Nardo LG, Hunter HR, et al. Cryopreserved-thawed embryo transfer in natural or downregulated hormonally controlled cycles: a retrospective study. Fertility and sterility. 2006;85(3):603-9.

12. Hancke K, More S, Kreienberg R, et al. Patients undergoing frozen-thawed embryo transfer have similar live birth rates in spontaneous and artificial cycles. J Assist Reprod Genet. 2012;29(5):403-407.

13. Tomas C, Alsbjerg B, Martikainen H, et al. Pregnancy loss after frozen-embryo transfer-a comparison of three protocols. Fertility and sterility 2012;98(5):1165-1169.

14. HillMJ, Miller KA, Frattarelli JL. A GnRH agonist and exogenous hormone stimulation protocol has a higher live-birth rate than a natural endogenous hormone protocol for frozen-thawed blastocyst-stage embryo transfer cycles: an analysis of 1391 cycles. Fertility and sterility. 2010;93(2):416-422.

15. Zheng Y, Li Z, XiongM, et al. Hormonal replacement treatment improves clinical pregnancy in frozen thawed embryos transfer cycles: a retrospective cohort study. Am J Transl Res 2014;6(1):85-90.

16. Cerrillo M, Herrero L, Guillen A, et al. Impact of endometrial preparation protocols for frozen embryo transfer on live birth rates. Rambam Maimonides medical journal. 2017; 8(2): e0020.

17. Givens CR, Markun LC, Ryan IP, et al. Outcomes of natural cycles versus programmed cycles for 1677 frozen-thawed embryo transfers. Reproductive biomedicine online. 2009; 19(3): 380-384.

18. Lyall F, Robson SC, Bulmer JN. Spiral artery remodeling and trophoblast invasion in preeclampsia and fetal growth restriction: relationship to clinical outcome. Hypertension 2013; 62(6): p. 1046-54.

19. Hiura H, Hattori H, Kobayashi N, et al. Genome-wide microRNA expression profiling in placentae from frozen-thawed blastocyst transfer. Clin Epigenetics. 2017; 9(3): p. 79.

20. Rizzo, G, Aiello E, Pietrolucci ME, et al. Are There Differences in Placental Volume and Uterine Artery Doppler in Pregnancies Resulting From the Transfer of Fresh Versus Frozen-Thawed Embryos Through In Vitro Fertilization. Reprod Sci. 2016; 23(10): p. 1381-6.

21. Fritz R, Jindal S, Feil H, et al. Elevated serum estradiol levels in artificial autologous frozen embryo transfer cycles negatively impact ongoing pregnancy and live birth rates. J Assist Reprod Genet. 2017; 34(12): 1633-1638.

22. Patel S, Kilburn B, Imudia A, et al. Estradiol elicits proapoptotic and antiproliferative effects in human trophoblast cells. Biol Reprod. 2015; 93(3): 74.

23. Shavit T, Oron G, Weon-Young S, et al. Vitrified-warmed single-embryo transfers may be associated with increased maternal complications compared with fresh single-embryo transfers. Reprod Biomed Online. 2017;35(1):94-102.

24. Sites CK, Wilson D, Barsky M, et al. Embryo cryopreservation and preeclampsia risk. Fertility and sterility. 2017; 108(5): 784-790.

Table 1. Baseline characteristics between different endometrial preparation groups

\begin{tabular}{lllll}
\hline Variable & Variable & $\mathrm{NC}(\mathrm{n}=1676)$ & $\mathrm{AC}(\mathrm{n}=8057)$ & $P$-value \\
\hline Female age at oocyte retrieval (y) & $31.19 \pm 4.86$ & $31.19 \pm 4.86$ & $30.46 \pm 4.92$ & $<0.001$ \\
Female age at embryo transfer (y) & $31.66 \pm 4.72$ & $31.66 \pm 4.72$ & $30.89 \pm 4.85$ & $<0.001$ \\
Infertility duration (y) & $3.43 \pm 2.97$ & $3.43 \pm 2.97$ & $3.47 \pm 2.88$ & 0.565 \\
$\mathrm{BMI}\left(\mathrm{kg} / \mathrm{m}^{2}\right)$ & $21.80 \pm 2.88$ & $21.80 \pm 2.88$ & $22.23 \pm 3.17$ & $<0.001$ \\
$<18.5(\mathrm{n}, \%)$ & $153(9.93)$ & $153(9.93)$ & $709(9.30)$ & \\
$18.5-25(\mathrm{n}, \%)$ & $1196(77.61)$ & $1196(77.61)$ & $5544(72.70)$ & \\
$>25(\mathrm{n}, \%)$ & $192(12.46)$ & $192(12.46)$ & $1373(18.00)$ & \\
AFC (n) & $10.91 \pm 5.15$ & $10.91 \pm 5.15$ & $12.97 \pm 6.07$ & $<0.001$ \\
No. of oocyte retrieved (n) & $11.98 \pm 6.69$ & $11.98 \pm 6.69$ & $13.78 \pm 7.58$ & $<0.001$ \\
Fertilization type (n, \%) & & & & 0.948 \\
$\quad$ IVF & $1148(73.45)$ & $1148(73.45)$ & $5681(73.65)$ & \\
$\quad$ ICSI & $370(23.67)$ & $370(23.67)$ & $1821(23.61)$ &
\end{tabular}




\begin{tabular}{|c|c|c|c|c|}
\hline Variable & Variable & $\mathrm{NC}(\mathrm{n}=1676)$ & $\mathrm{AC}(\mathrm{n}=8057)$ & $P$-value \\
\hline$\overline{I V F+I C S I ~}$ & $45(2.88)$ & $45(2.88)$ & $211(2.74)$ & \\
\hline Infertility type (n, \%) & & & & 0.220 \\
\hline Primary infertility & $842(54.50)$ & $842(54.50)$ & $4036(52.79)$ & \\
\hline Secondary infertility & $703(45.50)$ & $703(45.50)$ & $3609(47.21)$ & \\
\hline Protocol in the fresh cycle (n, \%) & & & & 0.004 \\
\hline Agonist & $1127(72.48)$ & $1127(72.48)$ & $5726(74.43)$ & \\
\hline Antagonist & $307(19.74)$ & $307(19.74)$ & $1537(19.98)$ & \\
\hline Other & $121(7.78)$ & $121(7.78)$ & $430(5.59)$ & \\
\hline Previous fresh embryo transfer $(n, \%)$ & $741(44.21)$ & $741(44.21)$ & $3858(47.88)$ & 0.006 \\
\hline Clinical pregnancy in fresh cycles $(\mathrm{n}, \%)$ & $427(57.6)$ & $427(57.6)$ & $2134(55.3)$ & 0.246 \\
\hline Single blastocyst transfer (n, \%) & & & & 0.764 \\
\hline No & $1343(80.13)$ & $1343(80.13)$ & $6482(80.45)$ & \\
\hline Yes & $333(19.87)$ & $333(19.87)$ & $1575(19.55)$ & \\
\hline Endometrial thickness on the day of $\mathrm{P}$ administration $(\mathrm{mm})$ & $10.94 \pm 1.93$ & $10.94 \pm 1.93$ & $9.98 \pm 1.55$ & $<0.001$ \\
\hline$<7$ & $21(1.25)$ & $21(1.25)$ & $137(1.70)$ & $? \dot{\imath} ?$ \\
\hline 7 & $1655(98.75)$ & $1655(98.75)$ & $7909(98.30)$ & \\
\hline Triple-line endometrial pattern (n, \%) & & & & 0.005 \\
\hline A & $284(16.96)$ & $284(16.96)$ & $1130(14.05)$ & \\
\hline B & $1274(76.06)$ & $1274(76.06)$ & $6388(79.41)$ & \\
\hline $\mathrm{C}$ & $117(6.99)$ & $117(6.99)$ & $526(6.54)$ & \\
\hline Type of embryo transferred (n, \%) & & & & 0.121 \\
\hline Cleavage stage & $752(44.87)$ & $752(44.87)$ & $3449(42.81)$ & \\
\hline Blastocyst stage & $924(55.13)$ & $924(55.13)$ & $4608(57.19)$ & \\
\hline No. of transferred embryos (n, \%) & & & & 0.219 \\
\hline 1 & $461(27.51)$ & $461(27.51)$ & $2126(26.39)$ & \\
\hline 2 & $1188(70.88)$ & $1188(70.88)$ & $5835(72.42)$ & \\
\hline 3 & $27(1.61)$ & $27(1.61)$ & $96(1.19)$ & \\
\hline No. of transferrable embryos (n) & $6.42 \pm 4.00$ & $6.42 \pm 4.00$ & $7.26 \pm 4.53$ & $<0.001$ \\
\hline No. of good quality embryos transferred (n, \%) & & & & 0.001 \\
\hline 0 & $660(39.40)$ & $660(39.40)$ & $2770(34.38)$ & \\
\hline 1 & $592(35.34)$ & $592(35.34)$ & $3051(37.87)$ & \\
\hline 2 & $423(25.25)$ & $423(25.25)$ & $2236(27.73)$ & \\
\hline
\end{tabular}

NC, natural cycle; AC, artificial cycle; BMI, body mass index; AFC, antral follicle count; IVF, in vitro fertilization; ICSI, intracytoplasmic sperm injection; P, progesterone.

Table 2. FET treatment and pregnancy outcomes in NC and AC cycles

\begin{tabular}{llll}
\hline & $\mathrm{NC}(\mathrm{n}=1676)$ & $\mathrm{AC}(\mathrm{n}=8057)$ & $P$-value \\
\hline Biochemical pregnancy (n, \%) & $1073(64.05)$ & $5347(66.36)$ & 0.076 \\
Implantation rate (n, \%) & $1384(47.43)$ & $6963(49.44)$ & 0.007 \\
Live birth rate (n, \%) & $885(52.80)$ & $4123(51.17)$ & 0.224 \\
Miscarriage rate (n, \%) & $118(11.47)$ & $794(15.85)$ & $<0.001$ \\
Clinical pregnancy rate (n, \%) & $1029(61.40)$ & $5009(62.09)$ & 0.553 \\
Preterm birth rate (n, \%) & $171(10.20)$ & $925(11.48)$ & 0.132 \\
Very preterm rate (<32 weeks) (n, \%) & $17(1.01)$ & $110(1.37)$ & 0.252 \\
Extremely preterm rate (<28 weeks) (n, \%) & $2(0.12)$ & $14(0.17)$ & 0.250 \\
Birth weight (kg) & $3.10 \pm 0.62$ & $3.10 \pm 0.65$ & 0.860 \\
Low birth weight (n, \%) & $165(9.84)$ & $817(10.14)$ & 0.715
\end{tabular}




\begin{tabular}{llll}
\hline & $\mathrm{NC}(\mathrm{n}=1676)$ & $\mathrm{AC}(\mathrm{n}=8057)$ & $P$-value \\
\hline Very low birth weight (n, \%) & $11(0.66)$ & $86(1.07)$ & 0.123 \\
Extremely low birth weight (n, \%) & $3(0.18)$ & $11(0.14)$ & 0.676 \\
Ectopic pregnancy rate (n, \%) & $10(0.60)$ & $47(0.58)$ & 0.948 \\
Fetal sex (n, \%) & & & 0.018 \\
Female & $433(48.98)$ & $1840(44.62)$ & \\
Male & $451(51.02)$ & $2284(55.38)$ & \\
\hline
\end{tabular}

NC, natural cycle; AC, artificial cycle.

Data presented as n (percent) or mean +/- standard deviation.

Table 3. Univariate analysis for live birth rate

\begin{tabular}{|c|c|c|c|}
\hline Covariate & Statistics & OR $(95 \% \mathrm{CI})$ & $P$-value \\
\hline Female age at oocyte retrieval (y) & $30.58 \pm 4.91$ & $0.92(0.91,0.92)$ & $<0.001$ \\
\hline Female age at embryo transfer (y) & $31.03 \pm 4.84$ & $0.92(0.91,0.92)$ & $<0.001$ \\
\hline Infertility duration $(\mathrm{y})$ & $3.46 \pm 2.89$ & $0.97(0.95,0.98)$ & $<0.001$ \\
\hline \multicolumn{4}{|l|}{$\operatorname{BMI}\left(\mathrm{kg} / \mathrm{m}^{2}\right)$} \\
\hline$<18.5(\mathrm{n}, \%)$ & $862(9.40 \%)$ & Reference & \\
\hline $18.5-25(\mathrm{n}, \%)$ & $6740(73.52 \%)$ & $0.96(0.83,1.10)$ & 0.553 \\
\hline$>25(\mathrm{n}, \%)$ & $3065(33.44 \%)$ & $0.77(0.65,0.91)$ & 0.003 \\
\hline $\mathrm{AFC}(\mathrm{n})$ & $12.63 \pm 5.98$ & $1.05(1.04,1.06)$ & $<0.001$ \\
\hline No. of oocyte retrieved (n) & $13.48 \pm 7.47$ & $1.05(1.04,1.05)$ & $<0.001$ \\
\hline \multicolumn{4}{|l|}{ Fertilization type $(\mathrm{n}, \%)$} \\
\hline IVF & $6829(73.62 \%)$ & Reference & \\
\hline ICSI & $2191(23.62 \%)$ & $1.08(0.98,1.19)$ & 0.106 \\
\hline IVF+ICSI & $256(2.76 \%)$ & $1.04(0.81,1.34)$ & 0.749 \\
\hline \multicolumn{4}{|l|}{ Infertility type (n, \%) } \\
\hline Primary infertility & $4878(53.08 \%)$ & Reference & \\
\hline Secondary infertility & $4312(46.92 \%)$ & $0.71(0.65,0.77)$ & $<0.001$ \\
\hline \multicolumn{4}{|l|}{ Protocol in the fresh cycle (n, \%) } \\
\hline Agonist & $6853(74.10 \%)$ & Reference & \\
\hline Antagonist & $1844(19.94 \%)$ & $0.57(0.52,0.64)$ & $<0.001$ \\
\hline Other & $551(5.96 \%)$ & $0.38(0.32,0.46)$ & $<0.001$ \\
\hline Previous fresh embryo transfer (n, \%) & $4599(47.23)$ & $0.81(0.73,0.96)$ & 0.082 \\
\hline Clinical pregnancy in fresh cycles $(\mathrm{n}, \%)$ & $2561(55.69)$ & $0.92(0.83,1.01)$ & 0.097 \\
\hline \multicolumn{4}{|l|}{ Single blastocyst transfer $(\mathrm{n}, \%)$} \\
\hline No & $7825(80.40)$ & Reference & \\
\hline Yes & $1908(19.60)$ & $0.74(0.67,0.82)$ & $<0.001$ \\
\hline Endometrial thickness on the day of $\mathrm{P}$ administration (mm) & $10.15 \pm 1.66$ & $1.07(1.04,1.09)$ & $<0.001$ \\
\hline \multicolumn{4}{|l|}{ Triple-line endometrial pattern (n, \%) } \\
\hline $\mathrm{A}$ & $1414(14.55)$ & Reference & \\
\hline $\mathrm{B}$ & $7662(78.84)$ & $0.91(0.81,1.02)$ & 0.102 \\
\hline $\mathrm{C}$ & $643(6.62)$ & $0.63(0.52,0.76)$ & $<0.001$ \\
\hline \multicolumn{4}{|l|}{ Type of embryo transferred (n, \%) } \\
\hline Cleavage stage & $4201(43.16)$ & Reference & \\
\hline Blastocyst stage & $5532(56.84)$ & $1.61(1.48,1.74)$ & $<0.001$ \\
\hline \multicolumn{4}{|l|}{ No. of transferred embryos (n, \%) } \\
\hline 1 & $2587(26.58)$ & Reference & \\
\hline 2 & $7023(72.16)$ & $1.80(1.64,1.97)$ & $<0.001$ \\
\hline
\end{tabular}




\begin{tabular}{llll}
\hline Covariate & Statistics & OR $(95 \% \mathrm{CI})$ & $P$-value \\
\hline 3 & $123(1.26)$ & $0.74(0.51,1.09)$ & 0.129 \\
No. of transferrable embryos $(\mathrm{n})$ & $7.08 \pm 4.34$ & $1.10(1.08,1.11)$ & $<0.001$ \\
No. of good quality embryos transferred $(\mathrm{n}, \%)$ & & & \\
0 & $3430(35.24)$ & Reference & \\
1 & $3643(37.43)$ & $1.68(1.53,1.85)$ & $<0.001$ \\
2 & $2659(27.30)$ & $2.89(2.60,3.22)$ & $<0.001$ \\
\hline
\end{tabular}

OR, odds ratio; CI, confidence interval; BMI, body mass index; AFC, antral follicle count; P, progesterone; IVF, in vitro fertilization; ICSI, intracytoplasmic sperm injection.

Table 4. Relationship between endometrial preparation and clinical outcomes in different models

\begin{tabular}{|c|c|c|c|c|}
\hline Outcome & Crude Model $^{\text {a }}$ & Crude Model $^{\text {a }}$ & Adjusted Model b & Adjusted Model $^{b}$ \\
\hline & OR $(95 \% \mathrm{CI})$ & $P$-value & OR $(95 \% \mathrm{CI})$ & $P$-value \\
\hline \multicolumn{5}{|l|}{ Live birth } \\
\hline $\mathrm{NC}$ & Reference & & Reference & \\
\hline $\mathrm{AC}$ & $0.94(0.84,1.04)$ & 0.224 & $0.89(0.79,1.01)$ & 0.055 \\
\hline \multicolumn{5}{|c|}{ Miscarriage } \\
\hline $\mathrm{NC}$ & Reference & & Reference & \\
\hline $\mathrm{AC}$ & $1.44(1.18,1.76)$ & 0.0003 & $1.38(1.11,1.73)$ & 0.004 \\
\hline \multicolumn{5}{|c|}{ Clinical pregnancy } \\
\hline $\mathrm{NC}$ & Reference & & Reference & \\
\hline $\mathrm{AC}$ & $1.03(0.93,1.15)$ & 0.553 & $0.96(0.85,1.09)$ & 0.540 \\
\hline \multicolumn{5}{|c|}{ Preterm birth } \\
\hline $\mathrm{NC}$ & Reference & & Reference & \\
\hline $\mathrm{AC}$ & $1.14(0.96,1.36)$ & 0.132 & $1.09(0.90,1.33)$ & 0.372 \\
\hline \multicolumn{5}{|c|}{ Ectopic pregnancy } \\
\hline $\mathrm{NC}$ & Reference & & Reference & \\
\hline $\mathrm{AC}$ & $0.98(0.49,1.94)$ & 0.95 & $0.77(0.36,1.61)$ & 0.484 \\
\hline
\end{tabular}

OR, odds ratio; CI, confidence interval; NC, natural cycle; AC, artificial cycle.

${ }^{a}$ We did not adjust other covariates.

b Adjusted for female age at embryo transfer, infertility duration, BMI, AFC, no. of oocyte retrieved, infertility type, protocol in the fresh cycle, single blastocyst transfer, endometrial thickness on the day of $\mathrm{P}$ administration, triple-line endometrial pattern, type of embryo transferred, no. of transferred embryos, no. of transferrable embryos and no. of good quality embryos transferred. 